\title{
AVALIAÇÃO DE LEGUMINOSAS FORRAGEIRAS (Centrosema spp.) EM SOLOS DISTRÓFICOS
}

\author{
N.J. NOVAES; R. GODOY; A.C.P.A. PRIMAVESI; G. de F. NEGREIROS \\ Centro de Pesquisa de Pecuária do Sudeste/EMBRAPA, C.P. 339, CEP: 13560-970 - São Carlos,SP
}

\begin{abstract}
RESUMO: Com a finalidade de avaliar a produtividade df acessos de Centrosema, previamente selecionados pelo CIAT para solos de baixa fertilidade, foi instalado na EMBRAPA-CPPSE de São Carlos, em Latossolo Vermelho-Amarelo, um ensaio em blocos ao acaso com quatro repetições, com 23 acessos de C.pubescens (2 testemunhas) e seis de C.acutifolium (2 testemunhas). A produção média de matéria seca dos seis cortes variou de 1240 a $2960 \mathrm{~kg} / \mathrm{ha}$ e os acessos que apresentaram as maiores produçōes, foram: Centrosema pubescens BRA 16918 e BRA 16985 e Centrosema acutifolium BRA 9211 e BRA 9229.

Deacritores: Centrosema, produtividade, adaptação, solos distroficos.
\end{abstract}

\section{EVALUATION OF FORAGE LEGUMES (Centrosema spp.) GROWN ON DISTROPIIC SOIIS}

ABSTRACT: With the purpose of evaluating forage yields of Centrosema accessions, which had been previously selected by CIAT to low fertility soils, an experiment was conducted at EMBRAPA-CPPSE, São Carlos,SP, on a Red-Yellow Latossol, using a randomized block design with four replications, and 23 accessions of C.pubescens (two controls) and 6 of C.acutifolium (two controls). Average dry matter yield of six harvests ranged from 1240 to 2960 $\mathrm{kg} / \mathrm{ha}$ and the best yielding accessions were C.pubescens: BRA 16918 and BRA 16985, and C.acutifolium: BRA 9211 and BRA 9229.

Key Words: Centrosema, yield, distrophicsoils, adaptation

\section{INTRODUÇÃO}

Centrosema pubescens ocupa importante lugar entre as leguminosas usadas para a formação de pastagens no Brasil tropical (DOBEREINER \& ARONOVICH, 1965). A planta possui folhas compostas, trifoliadas, inflorescências plurifloras, com flores violáceas ou brancas; sementes cilíndricas oblongas, marmoradas, negras quando secas (BARBOSA-FEVEREIRO, 1977) hábito de crescimento trepador, raiz pivotante bem desenvolvida $\mathrm{e}$ é perene (MONTEIRO, 1977). Desenvolve-se bem onde a precipitação média anual é maior que 1270 mm e a temperatura média está entre $22^{\circ} \mathrm{C}$ e $30^{\circ} \mathrm{C}$ (MATTOS, 1972). Essa espécie é considerada não adaptada a solos ácidos e de baixa fertilidade.

O presente trabalho teve por objetivo avaliar a adaptação e produção de matéria seca e de sementes de acessos de Centrosema previamente selecionados pelo CIAT, para solos de baixa fertilidade.

\section{MATERIAIS E MÉTODOS}

O ensaio foi instalado em 02/02/89, na EMBRAPA/Centro de Pesquisa de Pecuária do Sudeste, São Carlos,SP, situada a $22^{\circ} 01^{\prime} \mathrm{S}, 47^{\circ} 53^{\prime} \mathrm{W}$, com altitude de $856 \mathrm{~m}$ e média de precipitação anual de $1502 \mathrm{~mm}$, em Latossolo Vermelho-Amarelo, com características químicas apresentadas na TABELA 1, seguindo o delineamento de blocos ao acaso com quatro repetições.

Foram usados 23 acessos de Centrosema pubescens e 6 de C.acutifolium, conforme relação na TABELA 2.

A Figura 1 mostra as condições climáticas do período que o experimento foi conduzido.

As parcelas foram formadas por linhas de 10 plantas por acesso,com distância entre plantas de $0,25 \mathrm{~m}$, sendo o espaçamento entre a última planta de uma parcela e a primeira da próxima parcela de $1,5 \mathrm{~m}$ e a distância entre sulcos de $2 \mathrm{~m}$. Foram semeadas por cova, três sementes inoculadas com estirpe selecionada de Rhizobium. No plantio foi efetuada uma adubação fosfatada de $80 \mathrm{~kg} / \mathrm{ha}$ de $\mathrm{P}_{2} \mathrm{O}_{5}$, com superfosfato simples e um mês após uma aplicação em cobertura de $50 \mathrm{~kg} / \mathrm{ha}$ de $\mathrm{K}_{2} \mathrm{O}$, usando-se cloreto de potássio.

Em três blocos foram avaliados os seguintes parâmetros: produção de matéria seca em seis cortes efetuados em 13/11/89, 25/01/90, $25 / 04 / 90,26 / 11 / 90,14 / 03 / 91$ e 15/10/91, a capacidade de enraizamento, o crescimento dos ramos laterais e o desenvolvimento das plantas. No 
quarto bloco verificou-se o período de florescimento e a produção de sementes.

Para a avaliação de produção de matéria seca, foram realizadas amostragens em três locais de cada parcela com quadrado de $50 \mathrm{~cm}$ de lado, cortando-se as plantas a $5-10 \mathrm{~cm}$ do solo. Foi feito um corte de uniformização com o ensaio estabelecido (nove meses após a semeadura) e cortes para avaliações subsequentes no período chuvoso e no período seco. O material colhido foi seco em estufa a $65^{\circ} \mathrm{C}$ para a determinação do peso da matéria seca das amostras. $O$ crescimento dos ramos laterais foi determinado através de medições (tres de cada lado), a partir do centro da parcela, antes de cada corte. A avaliação de capacidade de enraizamento foi feita através de escala de notas de 0 (sem enraizamento) a 5 (maior enraizamento), antecedendo os cortes. $O$ desenvolvimento foi avaliado visualmente, usando-se uma escala de notas de 1 a 5 , em 18/08/89, 20/02/90, 23/04/90, $20 / 09 / 90,22 / 11 / 90,14 / 03 / 91$ e $15 / 10 / 91$.

\section{RESULTADOS}

Nas TABELAS 3 e 4 encontram-se os resultados de produção de matéria seca: dos seis cortes, da média de produção desses seis cortes, do periodo de um ano (de 1990 e de 1991) e das épocas da água e da seca desses dois anos.

A análise estatística dos dados de produção de matéria seca, referente a épocas de corte, indicou o primeiro corte como o de maior produção, provavelmente devido ao maior período de crescimento das plantas (fevereiro a novembro de 1989). Neste primeiro corte destacou-se como maior produtora de matéria seca a Centrosema pubescens BRA 16918 (5260kg/ha). O sexto e último corte apresentou a menor produção de matéria seca verificando-se que vários acessos não persistiram em alguns blocos (C.pubescens BRA 17043, BRA 17051 e BRA 10111 e C.acutifolium BRA 4162 e BRA 4821). Em estudos de crescimento e adaptação de espécies de Centrosema no sul da Flórida, verificou-se que os ecotipos também eram perenes de vida curta (KRETSCHMER, JR., 1977). As médias de produção de matéria seca dos seis cortes indicaram que os acessos de $C$. pubescens BRA 16918 e BRA 16985 e $C$. acutifolium BRA 9211 e BRA 9229, destacaram-se dos demais com $2960 \mathrm{~kg} / \mathrm{ha}, 2720 \mathrm{~kg} / \mathrm{ha}, 2660$ $\mathrm{kg} / \mathrm{ha}$ e $2610 \mathrm{~kg} / \mathrm{ha}$, respectivamente.

Os acessos de C.pubescens BRA 10111 (testemunha), BRA 17043, BRA 17051 e
C.acutifolium BRA 4162 (testemunha), apresentaram as menores médias de produção de matéria seca, onde a média geral obtida nos seis cortes foi de $2040 \mathrm{~kg} / \mathrm{ha}$.

No período de um ano $\left(1990=2^{\circ}+3^{\circ}\right.$ $+4^{\circ}$ cortes), destacaram-se os acessos: $C$. pubescens BRA 16985 e BRA 16918 e $C$. acutifolium BRA 9211 . $\mathrm{Na}$ época das águas $\left(2^{\circ}+\right.$ $3^{\circ}$ cortes): C.pubescens BRA 16985 e $C$. acutifolium BRA 4821 (testemunha) $\mathrm{e}$, na época seca (4 corte): $C$. acutifolium BRA 9211 e BRA 9229. No período de um ano $\left(1991=5^{\circ}+6^{\circ}\right.$ cortes), destacou-se C. acutifolium BRA 9229. Na época das águas: C. acutifolium BRA 9229 e BRA 4821 e, das águas, não houve diferença entre acessos. Na época da seca (1990) num período de 5 meses, a produção média obtida foi de 4719 $\mathrm{kg} / \mathrm{ha}$ e da seca ( 7 meses) de $1900 \mathrm{~kg} / \mathrm{ha}$ (40\% em relação ao período das águas) e em 11 meses do ano de 1991, num período de 4 meses (águas) a produção média obtida foi de $1733 \mathrm{~kg} / \mathrm{ha}$ e de 7 meses (seca) de $885 \mathrm{~kg} / \mathrm{ha}$ (51\% em relação ao período das águas. A redução na produção nos períodos de menor precipitação pluvial também foi verificada por HERNANDEZ et al. (1992).

Em um trabalho semelhante executado na Colombia (HERNANDEZ et al., 1992), destacaram-se os acessos de C.pubescens CIAT 5277 (testemunha), CIAT 5568 (testemunha), CIAT 15150, CIAT 15160 e CIAT 5172, com uma produção de matéria seca acumulada variando de 7,5 a 23,7 t/ha, e uma média de produção acumulada de seis cortes (1 ano e 4 meses) de 12,1 tha.

No presente trabalho, destacaram-se os acessos de C.pubescens CIAT 18180 (BRA 16918), CIAT 15160 (BRA 16985) e C.acutifolium CIAT 5112 (BRA 9211) e CIAT 15533 (BRA 9229) e a produção de matéria seca acumulada variou de 7,4 a 17,8 t/ha com uma média de produção acumulada de seis cortes ( 2 anos e 8 meses) de 12,2 t/ha.

PAYNE et al. (apud. SKERMAN, 1977), conseguiram, em Sigatoka, uma produção média de três anos de $4950 \mathrm{~kg} / \mathrm{ha}$ de matéria seca de Centrosema e DELISTOIANOV et al. (1987) produções de $1404 \mathrm{~kg} / \mathrm{ha}$ (média de 3 anos). A produção (média de 2 anos e 8 meses) neste experimento foi de $4600 \mathrm{~kg} / \mathrm{ha}$. WILSON \& LANSBURY (apud SKERMAN, 1977), relatam uma produção média anual de 13,5 t/ha de matéria verde. No Brasil, segundo SKERMAN (1977), tem sido observada uma produção média de $\mathbf{4 0}$ t/ha de matéria verde. 
TABELA 1 - Características químicas do solo.

\begin{tabular}{|c|c|c|c|c|c|c|c|c|c|c|c|c|}
\hline \multirow{2}{*}{$\begin{array}{l}\text { Prof. } \\
(\mathrm{cm}) \\
\end{array}$} & \multirow{2}{*}{$\begin{array}{c}\mathrm{pH} \\
\mathrm{CaCl}_{2} \\
\end{array}$} & \multirow{2}{*}{$\begin{array}{l}\text { P resina } \\
\text { (ug/cm })\end{array}$} & \multirow{2}{*}{$\begin{array}{c}\text { M.O. } \\
(\%)\end{array}$} & $\mathbf{K}$ & $\mathrm{Ca}$ & $\mathbf{M g}$ & $\mathrm{H}+\mathrm{Al}$ & Al & CTC & $s$ & $\mathrm{v}$ & $\mathbf{M}$ \\
\hline & & & & \multicolumn{7}{|c|}{$\mathrm{meq} / 100 \mathrm{~cm}^{3}$} & \multicolumn{2}{|c|}{$\%$} \\
\hline $0-30$ & 4,3 & 3 & 2,5 & 0,08 & 0,3 & 0,2 & 4,7 & 1,0 & 5,3 & 0,6 & 11 & 63 \\
\hline $30-60$ & 4,4 & 2 & 2,0 & 0,05 & 0,2 & 0,2 & 3,7 & 0,7 & 4,2 & 0,5 & 12 & 57 \\
\hline
\end{tabular}

Obs: Solo com $36 \%$ de argila, $1 \%$ de silte e $63 \%$ de areia.

TABELA 2 - Relação de acessos de Centrosema spp.

\begin{tabular}{|c|c|c|}
\hline Número CIAT & Cód. EMBRAPA & Espécie \\
\hline 15531 & BRA 009181 & Centrosema acutifolitum \\
\hline 5112 & BRA 009211 & Centrosema acutifolium \\
\hline 15533 & BRA 009229 & Centrosema acutifolium \\
\hline 15530 & BRA 009237 & Centrosema acutifolium \\
\hline 438 & BRA 002151 Test. & Centrosemasp \\
\hline 5596 & BRA 004537 & Centrosema pubescens \\
\hline 5189 & BRA 003191 & Centrosema pubescens \\
\hline 5277 & BRA 004162 Test. & Centrosema acutifolium \\
\hline 5568 & BRA 004821 Test. & Centrosema acmifolium \\
\hline 5627 & BRA 016896 & Centrosema pubescens \\
\hline 15150 & BRA 016918 & Centrosema pubescens. \\
\hline 413 & BRA 010111 Test. & Centrosemá pubescens. \\
\hline 15149 & BRA 016926 & Centrosema pubescens \\
\hline 15470 & BRA 016934 & Centrosema puhescens. \\
\hline 5172 & BRA 010227 & Centrosema pubescens \\
\hline 15154 & BRA 016942 & Cemrosema pubescens \\
\hline 15474 & BRA 016951 & Centrosema pubesce'ns \\
\hline 15043 & BRA 016696 & Centrosema pubescens \\
\hline 15144 & BRA 016977 & Centrosema pubescens \\
\hline 5169 & BRA 014559 & Centrosema pubescens \\
\hline 15160 & BRA 016985 & Centrosema pubescens \\
\hline 5133 & BRA 016993 & Centrosema pubescens. \\
\hline 5634 & BRA 017001 & Centrosema pubescens \\
\hline 5631 & BRA 017019 & Centrosema pubescens \\
\hline 5167 & BRA 017027 & Centrosema pubescens \\
\hline 15133 & BRA 017035 & Centrosema pubescens \\
\hline 5066 & BRA 014419 & Centrosema pubescens.s \\
\hline 15375 & BRA 017043 & Centrosema pubescens. \\
\hline 15132 & BRA 017051 & Centrosema pubescens \\
\hline
\end{tabular}


TABELA 3 - Produção de matéria seca (kg/ha) de Centrosema (média de 3 repetições).

\begin{tabular}{|c|c|c|c|c|c|c|c|}
\hline \multirow[b]{2}{*}{ Espécie } & \multirow[b]{2}{*}{$\begin{array}{l}\text { Código } \\
\text { EMBRA } \\
\text { PA } \\
\end{array}$} & \multicolumn{5}{|c|}{ Produção de Matéria Seca (kg/ha) } & \multirow[b]{2}{*}{$\begin{array}{l}\text { 6o corte } \\
(15.10 .91) \\
\text { Ep.Seca } \\
\end{array}$} \\
\hline & & $\begin{array}{l}\text { 10 corte } \\
(13.11 .89)\end{array}$ & $\begin{array}{l}20 \text { corte } \\
(25.02 .90) \\
\text { Ép.Água }\end{array}$ & $\begin{array}{l}\text { 3o corte } \\
(24.04 .90) \\
\text { Ép.Água }\end{array}$ & $\begin{array}{l}40 \text { corte } \\
(26.11 .90) \\
\text { Ép.Seca }\end{array}$ & $\begin{array}{l}\text { 5o corte } \\
(14.03 .91) \\
\text { Ép.Água }\end{array}$ & \\
\hline C.pubescens & 3191 & $2770 \mathrm{bc}$ & $2330 a$ & $2450 \mathrm{abc}$ & $650 c d$ & $1420 \mathrm{a}$ & $290 \mathrm{ab}$ \\
\hline C.pubescens & 4537 & $3260 \mathrm{bc}$ & $2300 a$ & $1720 \mathrm{abc}$ & $1020 \mathrm{bcd}$ & $1990 \mathrm{a}$ & $320 \mathrm{ab}$ \\
\hline C.pubescens & 10111 & $2300 \mathrm{bc}$ & $1850 a$ & $1290 c$ & $630 \mathrm{~cd}$ & $1150 \mathrm{a}$ & $190 \mathrm{ab}$ \\
\hline C.pubescens & (Test.) & $2260 \mathrm{bc}$ & $2610 a$ & $3060 \mathrm{ab}$ & 2130abcd & $1380 \mathrm{a}$ & $620 \mathrm{ab}$ \\
\hline C.pubescens & 10227 & $3040 b c$ & $2330 a$ & $3010 \mathrm{ab}$ & $2490 \mathrm{abcd}$ & $1730 a$ & $1250 \mathrm{ab}$ \\
\hline C.pubescens & 14419 & $3940 \mathrm{bc}$ & $3020 \mathrm{a}$ & $2650 \mathrm{abc}$ & $2460 \mathrm{abcd}$ & $2550 \mathrm{a}$ & $840 \mathrm{ab}$ \\
\hline C.pubescens & 14559 & $2970 \mathrm{bc}$ & $2160 \mathrm{a}$ & $2220 \mathrm{abc}$ & Id & $1510 \mathrm{a}$ & $880 \mathrm{ab}$ \\
\hline C.pubescens & 16896 & $5260 \mathrm{a}$ & $3370 a$ & $2370 \mathrm{abc}$ & $3340 \mathrm{abc}$ & $2130 \mathrm{a}$ & $1320 \mathrm{ab}$ \\
\hline C.pubescens & 16918 & $2720 b c$ & $2380 a$ & $2640 \mathrm{abc}$ & $1430 \mathrm{abcd}$ & $930 \mathrm{a}$ & $490 \mathrm{ab}$ \\
\hline C.pubescens & 16926 & $3040 b c$ & $2730 a$ & $2470 \mathrm{abc}$ & $3260 \mathrm{abc}$ & $1760 \mathrm{a}$ & $1250 \mathrm{ab}$ \\
\hline C.pubescens & 16934 & $3650 \mathrm{ab}$ & $2650 a$ & $2670 \mathrm{abc}$ & 2290abcd & $2030 \mathrm{a}$ & $1350 \mathrm{ab}$ \\
\hline C.pubescens & 16942 & $3100 \mathrm{bc}$ & $2660 \mathrm{a}$ & $2350 \mathrm{abc}$ & $2550 \mathrm{abcd}$ & $1680 \mathrm{a}$ & $1170 \mathrm{ab}$ \\
\hline C.pubescens & 16951 & $3760 \mathrm{ab}$ & $1970 a$ & $2250 \mathrm{abc}$ & 1330abcd & $2360 \mathrm{a}$ & $1100 \mathrm{ab}$ \\
\hline C.pubescens & 16969 & $2830 \mathrm{bc}$ & $1860 a$ & $2110 \mathrm{abc}$ & $2050 \mathrm{abcd}$ & $1700 \mathrm{a}$ & $520 \mathrm{ab}$ \\
\hline C.pubescens & 16977 & $3690 \mathrm{ab}$ & $2960 a$ & $3100 \mathrm{ab}$ & $3600 \mathrm{abc}$ & $1840 a$ & $1140 \mathrm{ab}$ \\
\hline C.pubescens & 16985 & $2510 \mathrm{bc}$ & $2020 a$ & $2280 \mathrm{abc}$ & $960 \mathrm{bcd}$ & $1530 \mathrm{a}$ & $340 \mathrm{ab}$ \\
\hline C.pubescens & 16993 & $3280 \mathrm{bc}$ & $2310 a$ & $2070 \mathrm{abc}$ & $2160 \mathrm{abcd}$ & $1990 \mathrm{a}$ & $1280 \mathrm{ab}$ \\
\hline C.pubescens & 17001 & $3020 b c$ & $2110 \mathrm{a}$ & $2030 \mathrm{abc}$ & $830 \mathrm{~cd}$ & $1390 \mathrm{a}$ & $560 \mathrm{ab}$ \\
\hline C.pubescens & 17019 & $2790 \mathrm{bc}$ & $2060 a$ & $1970 \mathrm{abc}$ & $2020 \mathrm{abcd}$ & $1770 \mathrm{a}$ & $910 \mathrm{ab}$ \\
\hline C.pubescens & 17027 & $2720 b c$ & $2150 a$ & $1530 \mathrm{bc}$ & $1780 \mathrm{abcd}$ & $1710 a$ & $1130 \mathrm{ab}$ \\
\hline C.pubescens & 17035 & $3210 b c$ & $2340 \mathrm{a}$ & $2050 \mathrm{abc}$ & $1 d$ & $1120 a$ & $190 a b$ \\
\hline C.pubescens & 17043 & $3050 \mathrm{bc}$ & $2070 a$ & $1820 \mathrm{abc}$ & Id & $880 a$ & $140 \mathrm{~b}$ \\
\hline C.acutifolium & 17051 & $1780 \mathrm{c}$ & $1770 \mathrm{a}$ & $3350 \mathrm{a}$ & $550 \mathrm{~cd}$ & $1421 \mathrm{a}$ & $400 \mathrm{ab}$ \\
\hline C.acutifolium & 4162 & $2950 \mathrm{bc}$ & $2600 a$ & $3240 a$ & $2100 \mathrm{abcd}$ & $1810 \mathrm{a}$ & $970 \mathrm{ab}$ \\
\hline C.acutifolitum & (Test.) & $2230 \mathrm{bc}$ & $1710 \mathrm{a}$ & $2320 \mathrm{abc}$ & $2360 \mathrm{abcd}$ & $1810 \mathrm{a}$ & $1360 \mathrm{ab}$ \\
\hline C.aculffolitum & 4821 & $3050 \mathrm{bc}$ & $2350 a$ & $2760 \mathrm{abc}$ & $4180 \mathrm{a}$ & $2180 a$ & $1420 \mathrm{ab}$ \\
\hline C.acutifolium & (Test.) & $2940 b c$ & $2300 \mathrm{a}$ & $2230 \mathrm{abc}$ & $4020 \mathrm{ab}$ & $2510 a$ & $1690 a$ \\
\hline C.acutifolium & 9181 & $2640 b c$ & $2480 a$ & $2810 \mathrm{abc}$ & $2560 \mathrm{abcd}$ & $2110 a$ & $1690 \mathrm{a}$ \\
\hline Centrosema & 9211 & $2990 \mathrm{bc}$ & $2400 a$ & $2140 \mathrm{abc}$ & $2370 \mathrm{abcd}$ & $1880 a$ & $850 a b$ \\
\hline \multirow[t]{4}{*}{ sp. } & 9229 & & & & & & \\
\hline & 9237 & & & & & & \\
\hline & 2151 & & & & & & \\
\hline & (Test.) & & & & & & \\
\hline CV (\%) & & 17,52 & 24.23 & 22,06 & 50,25 & 37,35 & 53,74 \\
\hline
\end{tabular}

Letras distintas indicam diferenças estatísticas na coluna (Tukey, $5 \%$ ) 
TABELA 4 - Produção de matéria de seca (kg/ha) de Centrosema (média de 3 repetições).

\begin{tabular}{|c|c|c|c|c|c|c|}
\hline Espécie & $\begin{array}{l}\text { Código } \\
\text { EMBRAPA }\end{array}$ & $\begin{array}{l}\text { Média } \\
\text { (6 cortes) }\end{array}$ & $\begin{array}{l}\text { Total } \\
\text { ( } 6 \text { cortes })\end{array}$ & $\begin{array}{l}1 \text { ano } 1990 \\
20+30+40 \\
\text { cortes }\end{array}$ & $\begin{array}{l}1 \text { ano } 1991 \\
50+60 \\
\text { cortes }\end{array}$ & $\begin{array}{l}\text { Época Águas } \\
20+30 \\
\text { cortes }\end{array}$ \\
\hline C.pubescens & 3191 & $1650 \mathrm{abcd}$ & $9910 \mathrm{abcd}$ & $5430 \mathrm{abc}$ & $1710 \mathrm{ab}$ & $4780 \mathrm{ab}$ \\
\hline C.pubescens & 4537 & 1770abcd & 10610abcd & $5040 \mathrm{abc}$ & $2310 \mathrm{ab}$ & $4020 \mathrm{ab}$ \\
\hline C.pubescens & 10111 (Test.) & $1240 \mathrm{~d}$ & $7410 \mathrm{~d}$ & $3780 \mathrm{c}$ & $1330 \mathrm{ab}$ & $3140 \mathrm{~b}$ \\
\hline C.pubescens & 10227 & 2010abcd & 12070abcd & $7800 \mathrm{abc}$ & $2010 \mathrm{ab}$ & $5670 \mathrm{ab}$ \\
\hline C.pubescens & 14419 & $2310 \mathrm{abcd}$ & $13850 \mathrm{abcd}$ & $7830 \mathrm{abc}$ & $2980 \mathrm{ab}$ & $5340 \mathrm{ab}$ \\
\hline C.pubescens & 14559 & $2530 \mathrm{abcd}$ & $15160 \mathrm{abcd}$ & $8130 \mathrm{abc}$ & $3100 \mathrm{ab}$ & $5670 \mathrm{ab}$ \\
\hline C.pubescens & 16896 & $1620 \mathrm{abcd}$ & $9750 \mathrm{abcd}$ & $4380 \mathrm{bc}$ & $2400 \mathrm{ab}$ & $4390 \mathrm{ab}$ \\
\hline C.pubescens & 16918 & $2960 \mathrm{a}$ & $17780 \mathrm{a}$ & $9070 \mathrm{ab}$ & $3450 \mathrm{ab}$ & $5730 \mathrm{ab}$ \\
\hline C.pubescens & 16926 & 1770abcd & 10590abcd & $6450 \mathrm{abc}$ & $1420 \mathrm{ab}$ & $5020 \mathrm{ab}$ \\
\hline C.pubescens & 16934 & $2420 \mathrm{abcd}$ & $14520 \mathrm{abcd}$ & $8470 \mathrm{abc}$ & $3010 \mathrm{ab}$ & $5210 \mathrm{ab}$ \\
\hline C.pubescens & 16942 & 2440abcd & $14640 \mathrm{abcd}$ & $7610 \mathrm{abc}$ & $3380 \mathrm{ab}$ & $5320 \mathrm{ab}$ \\
\hline C.pubescens & 16951 & $2250 \mathrm{abcd}$ & $13504 \mathrm{abcd}$ & $7560 \mathrm{abc}$ & $2860 \mathrm{ab}$ & $5010 \mathrm{ab}$ \\
\hline C.pubescens & 16969 & $2130 \mathrm{abcd}$ & $12770 \mathrm{abcd}$ & $5550 \mathrm{abc}$ & $3460 \mathrm{ab}$ & $4220 \mathrm{ab}$ \\
\hline C.pubescens & 16977 & $1850 \mathrm{abcd}$ & $11060 \mathrm{abcd}$ & $6020 \mathrm{abc}$ & $2210 \mathrm{ab}$ & $3970 \mathrm{ab}$ \\
\hline C.pubescens & 16985 & $2720 \mathrm{ab}$ & $16319 \mathrm{ab}$ & $9660 \mathrm{a}$ & $2980 \mathrm{ab}$ & $6060 \mathrm{a}$ \\
\hline C.pubescens & 16993 & $1610 \mathrm{abcd}$ & $9647 \mathrm{abcd}$ & $5260 \mathrm{abc}$ & $1880 \mathrm{ab}$ & $4300 \mathrm{ab}$ \\
\hline C.pubescens & 17001 & 2190abcd & $13100 \mathrm{abcd}$ & $6550 \mathrm{abc}$ & $3280 \mathrm{ab}$ & $4390 \mathrm{ab}$ \\
\hline C.pubescens & 17019 & $1660 \mathrm{abcd}$ & $9900 \mathrm{abcd}$ & $4970 a b c$ & $1950 \mathrm{ab}$ & $4140 a b$ \\
\hline C.pubescens & 17027 & 1920 abcd & $11522 \mathrm{abcd}$ & $6050 \mathrm{abc}$ & $2680 \mathrm{ab}$ & $4030 \mathrm{ab}$ \\
\hline C.pubescens & 17035 & $1840 \mathrm{abcd}$ & $11010 \mathrm{abcd}$ & $5450 \mathrm{abc}$ & $2840 \mathrm{ab}$ & $3680 \mathrm{ab}$ \\
\hline C.pubescens & 17043 & $1490 \mathrm{bcd}$ & $8900 \mathrm{bcd}$ & $4400 \mathrm{bc}$ & $1310 \mathrm{ab}$ & $4400 \mathrm{ab}$ \\
\hline C.pubescens & 17051 & $1330 \mathrm{~cd}$ & $7971 \mathrm{~cd}$ & $3890 \mathrm{c}$ & $1020 \mathrm{~h}$ & $3890 \mathrm{ab}$ \\
\hline C.acutifolumm & 4162 (Test.) & $1550 \mathrm{bcd}$ & $9300 \mathrm{bcd}$ & $5670 \mathrm{abc}$ & $1820 \mathrm{ab}$ & $5120 a b$ \\
\hline C.acutifolium & 4821 (Test.) & $2280 \mathrm{abcd}$ & $13684 a b c d$ & $7940 \mathrm{abc}$ & $2780 \mathrm{ab}$ & $58.50 \mathrm{a}$ \\
\hline C.acutifolium & 9181 & 1970abcd & $11790 \mathrm{abcd}$ & $6400 \mathrm{abc}$ & $3160 \mathrm{ab}$ & $4040 a b$ \\
\hline C.acutifolium & 9211 & $2660 \mathrm{abc}$ & 15940abc & $9300 a$ & $3600 \mathrm{ab}$ & $5110 a b$ \\
\hline C.acutifolium & 9229 & $2610 a b c$ & $15670 \mathrm{abc}$ & $8.540 \mathrm{abc}$ & $4200 a$ & $4520 a \mathrm{~h}$ \\
\hline C.acutfolium & 9237 & 2380abcd & $14290 \mathrm{abed}$ & $7860 \mathrm{abc}$ & $3800 \mathrm{ab}$ & $5290 \mathrm{ab}$ \\
\hline $\begin{array}{l}\text { Centrosema } \\
\mathrm{sp}\end{array}$ & 2151 (Test) & 2100abcd & 12610abcd & $6900 \mathrm{abc}$ & $2730 \mathrm{ab}$ & $4530 \mathrm{ab}$ \\
\hline$C V(\%)$ & & 20.68 & 20.71 & 23.02 & 34,74 & 17.33 \\
\hline
\end{tabular}

Letras distintas indicam diferenças estatístıcas na coluna (Tukey, 5\%) 
TABELA 5 - Produção de sementes e meses de florescimento.

\begin{tabular}{|c|c|c|c|c|c|c|}
\hline \multirow[t]{2}{*}{ Espécie } & \multirow{2}{*}{$\begin{array}{l}\text { Código } \\
\text { EMBRAPA }\end{array}$} & \multirow{2}{*}{$\frac{\text { Meses de }}{1990}$} & \multirow{2}{*}{$\begin{array}{c}\text { Florescimento } \\
1991\end{array}$} & \multirow{2}{*}{$\frac{\text { Produção }}{1989}$} & \multirow{2}{*}{$\frac{\mathrm{de}}{1990}$} & \multirow{2}{*}{$\frac{\text { Sementes }(\mathrm{kg} / \mathrm{ha})}{1991}$} \\
\hline & & & & & & \\
\hline C.pubescens & 3191 & 4 a 6 & 4 a 9 & 62 & 4 & 15 \\
\hline C.pubescens & 4537 & 4 a 5 & 5 a 8 & 4 & 0 & 1 \\
\hline C.pubescens & 10111 (Test.) & 4 a 6 & 5 a 8 & 88 & 0 & 5 \\
\hline C.pubescens & 10227 & 4 a 6 & 4 a 8 & 4 & 154 & 70 \\
\hline C.pubescens & 14419 & 4 a 6 & 5 a 9 & 20 & 118 & 118 \\
\hline C.pubescens & 14559 & 4 a 6 & 4 a 8 & 6 & 134 & 87 \\
\hline C.pubescens & 16896 & 4 a 6 & 5 a 8 & 6 & 24 & 51 \\
\hline C.pubescens & 16918 & 4 a 6 & 5 a 9 & 6 & 44 & 242 \\
\hline C.pubescens & 16926 & 4 a 6 & 4 a 8 & 12 & 18 & 3 \\
\hline C.pubescens & 16934 & 4 a 6 & 5 a 8 & 24 & 374 & 51 \\
\hline C.pubescens & 16942 & 4 a 6 & 5 a 8 & 10 & 314 & 298 \\
\hline C.pubescens & 16951 & 4 a 6 & 5 a 9 & 4 & 494 & 211 \\
\hline C.pubescens & 16969 & 4 a 6 & 5 a 8 & 2 & 170 & 58 \\
\hline C.pubescens & 16977 & 3 a 6 & 5 a 8 & 16 & 66 & $9 \mathrm{I}$ \\
\hline C.pubescens & 16985 & 4 a 6 & 4 a 8 & 8 & 156 & 45 \\
\hline C.pubescens & 16993 & 3 a 6 & 3 a 8 & 10 & 18 & 128 \\
\hline C.pubescens & 17001 & 4 a 6 & 5 a 8 & 34 & 14 & 2 \\
\hline C.pubescens & 17019 & 5 a 6 & 5 a 8 & 4 & 0 & 1 \\
\hline C.pubescens & 17027 & 4 a 6 & 4 a 9 & 44 & 190 & 144 \\
\hline C.pubescens & 17035 & 4 a 6 & 4 a 8 & 2 & 2 & 4 \\
\hline C.pubescens & 17043 & 4 a 6 & 5 a 8 & 32 & 28 & 70 \\
\hline C.pubescens & 17051 & 4 a 6 & 5 a 8 & 2 & 6 & 132 \\
\hline C.acutifolium & 4162 (Test.) & 4 a 5 & 4 a 9 & 0 & 2 & 1 \\
\hline C. actutifolium & 4821 (Test.) & 4 a 6 & 4 a 8 & 2 & 2 & 12 \\
\hline C. acutifolinm & 9181 & 3 a 6 & 5 a 8 & 0 & 10 & 75 \\
\hline C.acutifolinm & 9211 & 4 a 6 & 4 a 8 & 2 & 66 & 94 \\
\hline C.actutifolium & 9229 & 4 a 6 & 4 a 8 & 2 & 4 & 38 \\
\hline C.acutifolium & 9237 & 4 a 6 & 4 a 8 & 2 & 0 & 25 \\
\hline Centrosema sp. & 2151 (Test.) & 4 a 6 & 5 a 8 & 0 & 0 & 1 \\
\hline
\end{tabular}


TABELA 6 - Crescimento lateral médio (cm) de acessos de Centrosema spp.

\begin{tabular}{|c|c|c|c|c|c|c|c|}
\hline \multirow[b]{2}{*}{ Espécie } & \multirow{2}{*}{$\begin{array}{l}\text { Código } \\
\text { EMBRAPA }\end{array}$} & \multicolumn{6}{|c|}{ Avaliaçōes } \\
\hline & & 13.11 .89 & 25.02 .90 & 25.04 .90 & 26.11 .90 & 14.03 .91 & 15.10 .91 \\
\hline C.pubescens & 16918 & 60.61 abcde & $55,56 \mathrm{ab}$ & $77.61 \mathrm{ab}$ & $58.00 \mathrm{abc}$ & $66.00 \mathrm{a}$ & $16.00 \mathrm{abcd}$ \\
\hline C.pubescens & 14559 & $51,22 \mathrm{cde}$ & $51.11 \mathrm{ab}$ & $59,56 \mathrm{ab}$ & $37.00 \mathrm{c}$ & $54.33 \mathrm{a}$ & $17,77 \mathrm{abcd}$ \\
\hline C.pubescens & 16969 & $51,00 \mathrm{cde}$ & $60,56 \mathrm{ab}$ & $73,05 \mathrm{ab}$ & $28,33 \mathrm{c}$ & $62,67 a$ & $17,33 \mathrm{abcd}$ \\
\hline C.pubescens & 16985 & $47,33 \mathrm{cde}$ & $60,00 \mathrm{ab}$ & $72,19 \mathrm{ab}$ & $42,33 \mathrm{abc}$ & $50,00 \mathrm{a}$ & $19.33 \mathrm{abcd}$ \\
\hline C.pubescens & 16942 & 54,94 abcde & $52.78 \mathrm{ab}$ & $64.33 \mathrm{ab}$ & $39,33 b c$ & $58,00 \mathrm{a}$ & $18.00 \mathrm{abcd}$ \\
\hline C.pubescens & 17001 & $72,61 a b c$ & $58.86 \mathrm{ab}$ & $69.61 \mathrm{ab}$ & $47,33 \mathrm{abc}$ & $68.00 \mathrm{a}$ & $22.33 \mathrm{abed}$ \\
\hline C.pubescens & 4537 & 61,61 abcde & $53,88 \mathrm{ab}$ & $61.39 \mathrm{ab}$ & $29,67 \mathrm{c}$ & $47,50 \mathrm{a}$ & $9.67 \mathrm{abcd}$ \\
\hline C.pubescens & 17043 & $63,61 \mathrm{abcd}$ & $58,33 \mathrm{ab}$ & $78.37 \mathrm{ab}$ & $14,50 \mathrm{c}$ & $18,00 \mathrm{a}$ & $3.33 \mathrm{~cd}$ \\
\hline C.pubescens & 16951 & $49,22 \mathrm{cde}$ & $57,22 \mathrm{ab}$ & $80,33 \mathrm{ab}$ & $51,33 \mathrm{abc}$ & $69,67 a$ & $17,67 \mathrm{abcd}$ \\
\hline C.pubescens & 17051 & 60.39abcde & $49,44 \mathrm{ab}$ & $54.28 \mathrm{ab}$ & $15,50 c$ & $30.15 \mathrm{a}$ & $1.67 \mathrm{~d}$ \\
\hline C.acutifolitum & 9211 & $92,39 \mathrm{a}$ & $71.67 \mathrm{a}$ & $84.38 \mathrm{ab}$ & $85,33 \mathrm{a}$ & $55.67 a$ & $27.33 \mathrm{ab}$ \\
\hline C.pubescens & 16934 & $55,56 \mathrm{cde}$ & $59,44 \mathrm{ab}$ & $84.55 \mathrm{ab}$ & $43,66 a b c$ & $52,67 a$ & $17,33 \mathrm{abcd}$ \\
\hline C.pubescens & 14419 & $67.72 \mathrm{abcd}$ & $55.56 \mathrm{ab}$ & $80.72 \mathrm{ab}$ & $44.66 \mathrm{abc}$ & $66.67 a$ & 23,00 abcd \\
\hline C.pubescens & 17019 & $64,61 \mathrm{abcd}$ & $55,56 \mathrm{ab}$ & $72.11 \mathrm{ab}$ & $22.33 \mathrm{c}$ & $71.50 \mathrm{a}$ & 14.33abcd \\
\hline Centrosema sp. & 2151 (Test.) & $49.44 \mathrm{cde}$ & $50,00 \mathrm{ab}$ & $55.72 \mathrm{ab}$ & $32.33 \mathrm{c}$ & $49.00 \mathrm{a}$ & $11.67 \mathrm{abcd}$ \\
\hline C.pubescens & 16896 & $58,89 \mathrm{abcde}$ & $50,00 \mathrm{ab}$ & $69.39 \mathrm{ab}$ & $17.67 \mathrm{c}$ & $70,00 \mathrm{a}$ & $14,67 \mathrm{abcd}$ \\
\hline C.acutifolium & 4821 (Test.) & $79,05 \mathrm{abc}$ & $57.78 \mathrm{ab}$ & $64.11 \mathrm{ab}$ & $31.50 \mathrm{c}$ & $29.00 \mathrm{a}$ & $12.33 \mathrm{abcd}$ \\
\hline C.acutifolum & 9229 & $91.33 \mathrm{a}$ & $72.78 \mathrm{a}$ & $89.33 a$ & $83.66 \mathrm{a}$ & $47.33 a$ & $25.00 \mathrm{abc}$ \\
\hline C.pubescens & 16977 & $57.56 \mathrm{abcde}$ & $57.22 \mathrm{ab}$ & $78.50 \mathrm{ab}$ & $44,33 \mathrm{abc}$ & $61.67 \mathrm{a}$ & $12.33 \mathrm{abed}$ \\
\hline C.pubescens & 17027 & $50.61 \mathrm{de}$ & $53.33 \mathrm{ab}$ & $66.22 \mathrm{ab}$ & $45.33 \mathrm{abc}$ & $56.00 \mathrm{a}$ & 14.00 abed \\
\hline C.pubescens & 3191 & $55.56 \mathrm{cde}$ & $51.11 \mathrm{ab}$ & $84.33 \mathrm{ab}$ & 28.67 & $62.00 \mathrm{a}$ & $5.33 \mathrm{bed}$ \\
\hline C.pubescens & 16926 & $54,61 \mathrm{cde}$ & $54.44 \mathrm{ab}$ & $63.28 \mathrm{ab}$ & $27.6 \%$ & $65.00 \mathrm{a}$ & $7.00 \mathrm{bud}$ \\
\hline$C$ pubescens & 17035 & 48.17 de & $47.78 \mathrm{ab}$ & $61.89 \mathrm{ab}$ & $28.33 c$ & $64.00 \mathrm{a}$ & 14.00abed \\
\hline C.acutifolium & 9237 & $90,11 \mathrm{ab}$ & $62.78 \mathrm{ab}$ & $82.55 \mathrm{ab}$ & $82.66 \mathrm{ab}$ & $53.33 \mathrm{a}$ & $30.00 \mathrm{a}$ \\
\hline C.pubescens & 16993 & $60.39 \mathrm{abcde}$ & $47.78 \mathrm{ab}$ & $73.05 \mathrm{ab}$ & $32.33 c$ & $55.5 .5 \mathrm{a}$ & $5.00 \mathrm{hed}$ \\
\hline C.pubescens & 10111 (Test) & $66.94 \mathrm{abcd}$ & $36.67 \mathrm{~h}$ & $46.44 b$ & $25,50 \mathrm{c}$ & $75.00 \mathrm{a}$ & $4,67 \mathrm{hed}$ \\
\hline Cpubescens & 10227 & $37,89 \mathrm{bc}$ & $43.89 \mathrm{ab}$ & $70.78 \mathrm{ab}$ & $29.00 c$ & $61.67 \mathrm{a}$ & 13.67ahed \\
\hline C.acutifolium & 9181 & $75,11 \mathrm{abc}$ & $62.78 \mathrm{ab}$ & $88.94 a$ & $52,00 \mathrm{abc}$ & $59.33 \mathrm{a}$ & $24.00 \mathrm{abcd}$ \\
\hline C.acutifolum & 4162 (Test.) & $27.94 \mathrm{e}$ & $35.56 \mathrm{~b}$ & $68.38 \mathrm{ab}$ & $16.50 \mathrm{c}$ & $55.00 \mathrm{a}$ & S.00bed \\
\hline $\mathrm{CV}(\%)$ & & 17.34 & 16.84 & 18.16 & 33,84 & 19.91 & 49.15 \\
\hline
\end{tabular}


TABELA 7 - Enraizamento médio dos ramos laterais de Centrosema spp.

\begin{tabular}{|c|c|c|c|c|c|c|c|}
\hline \multirow[b]{2}{*}{ Espécic } & \multirow{2}{*}{$\begin{array}{l}\text { Código } \\
\text { EMBRAPA }\end{array}$} & \multicolumn{5}{|c|}{ Avaliaçōes } & \multirow[b]{2}{*}{15.10 .91} \\
\hline & & 13.11 .89 & 25.02 .90 & 25.04 .90 & 26.11 .90 & 14.03 .91 & \\
\hline C.pubescens & 16918 & $1,00 \mathrm{a}$ & $2,00 \mathrm{a}$ & $1,67 \mathrm{gh}$ & $2,33 \mathrm{ab}$ & $2,67 \mathrm{a}$ & 0 \\
\hline C.pubescens & 14559 & $1,33 a$ & $2,67 a$ & 3,00 bcdef & $1,33 a b c$ & $2,33 \mathbf{a}$ & 0 \\
\hline C.pubescens & 16969 & $1,67 \mathrm{a}$ & $2,33 a$ & $3,00 \mathrm{bcdef}$ & $1,00 a b c$ & $2,00 \mathrm{a}$ & 0 \\
\hline C.pubescens & 16985 & $1,67 \mathrm{a}$ & $3,00 \mathrm{a}$ & $2,00 \mathrm{cdef}$ & $1,67 \mathrm{abc}$ & $1,33 a$ & 0 \\
\hline C.pubescens & 16942 & $1,33 \mathrm{a}$ & $2,33 a$ & $2,33 \mathrm{bcdef}$ & $1,67 a b c$ & $2.33 \mathrm{a}$ & 0 \\
\hline C.pubescens & 17001 & $1,67 \mathrm{a}$ & $3.00 \mathrm{a}$ & $3.33 \mathrm{abcde}$ & $1.67 \mathrm{abc}$ & $2,33 a$ & 0 \\
\hline C.pubescens & 4537 & $1,67 a$ & $2,67 \mathrm{a}$ & $1,67 \mathrm{def}$ & $0,67 b c$ & $2,00 \mathrm{a}$ & 0 \\
\hline C.pubescens & 17043 & $1,67 \mathrm{a}$ & $2,33 \mathrm{a}$ & $2.67 \mathrm{bcdef}$ & $0,00 \mathrm{c}$ & $2.00 \mathrm{a}$ & 0 \\
\hline C.pubescens & 16951 & $1,67 \mathrm{a}$ & $2,33 \mathbf{a}$ & $4,00 \mathrm{abc}$ & $2,33 \mathrm{ab}$ & $3.00 \mathrm{a}$ & 0 \\
\hline C.pubescens & 17051 & $1.67 \mathrm{a}$ & $3.00 \mathrm{a}$ & 3.67abcd & $0.00 c$ & $1,50 \mathrm{a}$ & 0 \\
\hline C.aculifolinm & 9211 & $2,33 \mathbf{a}$ & $3,00 \mathbf{a}$ & $4.00 \mathrm{abc}$ & $3.67 \mathrm{a}$ & $2.00 \mathrm{a}$ & 0 \\
\hline C.pubescens & 16934 & $1,67 \mathrm{a}$ & $2,67 \mathrm{a}$ & $4.00 \mathrm{abc}$ & $2,00 \mathrm{abc}$ & $2,67 \mathrm{a}$ & 0 \\
\hline C.pubescens & 14419 & $2,00 \mathbf{a}$ & $3.00 \mathrm{a}$ & $5.00 \mathrm{a}$ & $2,00 \mathrm{abc}$ & $2.33 \mathrm{a}$ & 0 \\
\hline C.pubescens & 17019 & $2,00 \mathrm{a}$ & $3,00 \mathrm{a}$ & 3,33abcde & $0.33 b c$ & $1,67 \mathrm{a}$ & 0 \\
\hline Centrosema sp. & 2151 (Test.) & $1,33 \mathbf{a}$ & $2,33 \mathrm{a}$ & $2.00 \mathrm{cdef}$ & $1,67 \mathrm{abc}$ & $1,33 \mathrm{a}$ & 0 \\
\hline C.pulescens & 16896 & $1.00 \mathrm{a}$ & $2.67 \mathrm{a}$ & $3,67 a b c d$ & $0.00 c$ & $1.67 \mathrm{a}$ & 0 \\
\hline C.aculyfolutum & 4821 (Test) & $2.67 \mathrm{a}$ & $2.67 \mathrm{a}$ & 3,00abcdef & $2,00 \mathrm{abc}$ & $1.00 \mathrm{a}$ & 0 \\
\hline C.acutifolmom & 9229 & $3.00 \mathrm{a}$ & $2,67 \mathrm{a}$ & 3.00abcdef & $4.00 \mathrm{a}$ & $2.67 \mathrm{a}$ & 0 \\
\hline C.pubescens & 16977 & $1.33 \mathrm{a}$ & $2,00 \mathrm{a}$ & $4.00 \mathrm{abc}$ & $1.67 \mathrm{abc}$ & $1,67 \mathrm{a}$ & 0 \\
\hline Cpubescens & 17027 & $1.33 \mathrm{a}$ & $2.33 \mathrm{a}$ & $2.00 \mathrm{cdef}$ & $1,67 \mathrm{abc}$ & $2.67 \mathrm{a}$ & 0 \\
\hline C.pubescens & $3[9]$ & $1.67 \mathrm{a}$ & $2.33 \mathrm{a}$ & $4.33 \mathrm{ab}$ & $1.00 \mathrm{abc}$ & $1.67 \mathrm{a}$ & 0 \\
\hline cipubescens & 16926 & $1.33 \mathrm{a}$ & $2.67 \mathrm{a}$ & $2.00 \mathrm{cdef}$ & $0.67 \mathrm{bc}$ & $1.67 \mathrm{a}$ & 0 \\
\hline C.pubcscens & 17035 & $1.67 \mathrm{a}$ & $2.33 \mathrm{a}$ & $1.67 \mathrm{def}$ & $0.67 \mathrm{bc}$ & $2.33 \mathrm{a}$ & 0 \\
\hline C.actulyfolium & 9237 & $2.33 a$ & $2.67 \mathrm{a}$ & $1.67 \mathrm{def}$ & $3.67 \mathrm{a}$ & $1.33 \mathrm{a}$ & 0 \\
\hline C.pubescens & 16993 & $2.00 \mathrm{a}$ & $2.67 \mathrm{a}$ & $2.67 \mathrm{bcdef}$ & $0,67 b c$ & $2.00 \mathrm{a}$ & 0 \\
\hline C.pubescens & 10111 (Test.) & $2.00 \mathrm{a}$ & $2.00 \mathrm{a}$ & $1.33 \mathrm{ef}$ & $0.50 \mathrm{bc}$ & $1.00 \mathrm{a}$ & 0 \\
\hline C.pubescens & 10227 & $1,00 \mathrm{a}$ & $2.00 \mathrm{a}$ & $2,67 \mathrm{bcdef}$ & $1.00 \mathrm{abc}$ & $2.00 \mathrm{a}$ & 0 \\
\hline C.acutyfolium & 9181 & $3,00 \mathrm{a}$ & $3,00 \mathrm{a}$ & $3.00 \mathrm{abcdef}$ & $2,00 \mathrm{abc}$ & $1.67 \mathrm{a}$ & 0 \\
\hline C. acutifolium & 4162 (Test.) & $1,00 \mathrm{a}$ & $1.67 \mathrm{a}$ & $1.00 \mathrm{f}$ & $0.50 \mathrm{bc}$ & $2.00 \mathrm{a}$ & 0 \\
\hline $\mathrm{CV}(\%)$ & & 29.16 & 8.33 & 10.63 & 21.89 & 19.26 & \\
\hline
\end{tabular}

Letras distintas indicam dıferunças estatísticas na coluna (Tukey. 5\%). 
TABELA 8 - Avaliação visual (média de 3 repetições) de desenvolvimento de Centrosema spp. (1 = pouco desenvolvimento; 5 = desenvolvimento máximo).

\begin{tabular}{|c|c|c|c|c|c|c|c|c|c|}
\hline \multirow[b]{2}{*}{ Espécie } & \multirow{2}{*}{$\begin{array}{l}\text { Código } \\
\text { EM- } \\
\text { BRAPA }\end{array}$} & \multicolumn{8}{|c|}{ Avalıaçōes } \\
\hline & & $\begin{array}{l}18 / 08 / \\
1989\end{array}$ & $\begin{array}{l}17 / 10 / \\
1989\end{array}$ & $\begin{array}{l}20 / 12 / \\
1989\end{array}$ & $\begin{array}{l}23 / 04 / \\
1990\end{array}$ & $\begin{array}{l}20 / 09 / \\
1990\end{array}$ & $\begin{array}{l}22 / 11 / \\
1990\end{array}$ & $\begin{array}{l}14 / 03 \\
/ 1991\end{array}$ & $\begin{array}{l}15 / 10 / \\
1991\end{array}$ \\
\hline C.pubescens & 16918 & $4.76 \mathrm{a}$ & $5,00 \mathrm{a}$ & $3,67 \mathrm{abc}$ & $3,67 \mathrm{abcd}$ & $3,00 \mathrm{abc}$ & $3,67 \mathrm{ab}$ & $2,67 \mathrm{a}$ & $4,00 \mathrm{ab}$ \\
\hline C.pubescens & 14559 & $4,33 \mathrm{ab}$ & $3,67 \mathrm{ab}$ & $3,67 \mathrm{ab}$ & $4,00 \mathrm{abc}$ & $1,67 \mathrm{~cd}$ & $2,67 \mathrm{ab}$ & $2,00 a$ & $3,00 \mathrm{ab}$ \\
\hline C.pubescens & 16969 & 3,00abcde & 3,33abc & $4,00 \mathrm{abc}$ & 2,67abcd & $1,67 \mathrm{~cd}$ & $2,33 \mathrm{ab}$ & $1,67 \mathrm{a}$ & $3,67 \mathrm{ab}$ \\
\hline C.pubescens & 16985 & 2,67 abcde & $3,33 \mathrm{abc}$ & $4.00 \mathrm{abc}$ & $4.00 \mathrm{abc}$ & $3,00 \mathrm{abc}$ & $3,00 \mathrm{ab}$ & $2,33 a$ & $3,33 \mathrm{ab}$ \\
\hline C.pubescens & 16942 & $2,67 a b c d e$ & $3,67 \mathrm{ab}$ & $3,00 \mathrm{abc}$ & $3,33 \mathrm{abcd}$ & $2,33 \mathrm{abcd}$ & $3,00 \mathrm{ab}$ & $2,67 a$ & $3,67 \mathrm{ab}$ \\
\hline C.pubescens & 17001 & $4,00 \mathrm{abc}$ & 4,33ab & $4.00 \mathrm{abc}$ & 2,33abcd & $1.67 \mathrm{ed}$ & $3,00 \mathrm{ab}$ & $2,00 \mathrm{a}$ & $3.33 \mathrm{ab}$ \\
\hline C.pubescens & 4537 & 2,67 abcde & $3,00 \mathrm{abc}$ & $4.00 \mathrm{abc}$ & $1.67 \mathrm{~cd}$ & $1.00 \mathrm{~cd}$ & $2.00 \mathrm{ab}$ & $1,33 \mathrm{a}$ & $2,33 \mathrm{ab}$ \\
\hline C.pubescens & 17043 & $4,00 \mathrm{abc}$ & $4.00 \mathrm{ab}$ & $4.00 \mathrm{abc}$ & $2,00 \mathrm{bcd}$ & $0.67 \mathrm{~cd}$ & $1,00 \mathrm{~b}$ & $1.55 \mathrm{a}$ & $1,67 \mathrm{ab}$ \\
\hline C.pubescens & 16951 & 2,67 abcde & $3.67 \mathrm{ab}$ & 3,33abc & $3,33 \mathrm{abcd}$ & $2.33 \mathrm{abcd}$ & $3.33 \mathrm{ab}$ & $3.67 \mathrm{a}$ & $4.00 \mathrm{ab}$ \\
\hline C.pubescens & 17051 & 2,67 abcde & $3,67 \mathrm{ab}$ & $4,33 \mathrm{ab}$ & $2,00 \mathrm{bcd}$ & $0,67 \mathrm{~d}$ & $1,00 \mathrm{~b}$ & $1.50 \mathrm{a}$ & $0.67 \mathrm{~h}$ \\
\hline C. acutfollum & 9211 & $4.33 \mathrm{ab}$ & $4,33 \mathrm{ab}$ & $4.33 \mathrm{ab}$ & $4,33 \mathrm{ab}$ & $5.00 \mathrm{a}$ & $5.00 \mathrm{a}$ & $3.67 \mathrm{a}$ & $4.67 \mathrm{a}$ \\
\hline C.pubescens & 16934 & 2,67 abcde & $3.67 \mathrm{ah}$ & $3.33 \mathrm{abc}$ & $3,00 \mathrm{abcd}$ & $3,00 \mathrm{abc}$ & $3.67 \mathrm{ab}$ & $2.67 \mathrm{a}$ & $4.33 \mathrm{a}$ \\
\hline C.pubescens & 14419 & $2,67 \mathrm{abcde}$ & $3,00 \mathrm{abc}$ & $4.00 \mathrm{abc}$ & $2.33 \mathrm{abcd}$ & $2,67 \mathrm{abcd}$ & $3,33 \mathrm{ab}$ & $2.67 a$ & $4.33 \mathrm{a}$ \\
\hline C.pubescens & 17019 & $3.33 \mathrm{abcd}$ & $3.33 \mathrm{abc}$ & $3.33 \mathrm{abc}$ & $2.00 \mathrm{bcd}$ & $1.00 \mathrm{~cd}$ & $2.00 \mathrm{ab}$ & $1,33 a$ & $2.67 \mathrm{ah}$ \\
\hline Centrosema sp & 2151 & 2,33abcde & $2,67 \mathrm{abc}$ & $2.67 \mathrm{abc}$ & 3,33abcd & $2.00 \mathrm{bed}$ & $3,67 \mathrm{ab}$ & $2.33 \mathrm{a}$ & $3.33 \mathrm{ab}$ \\
\hline C.pubescens & (Test.) & $2.00 \mathrm{cde}$ & $3,33 \mathrm{abc}$ & $2.67 \mathrm{abc}$ & $2.67 \mathrm{abcd}$ & $1,00 \mathrm{ed}$ & $2,00 \mathrm{ab}$ & $2,00 \mathrm{a}$ & $2.67 \mathrm{ah}$ \\
\hline C. acuifolium & 16896 & $4,00 \mathrm{abc}$ & $3,00 \mathrm{abc}$ & $4,33 \mathrm{ah}$ & $3,67 \mathrm{abcd}$ & $1,67 \mathrm{~cd}$ & $4,00 \mathrm{ab}$ & $2,50 \mathrm{a}$ & $3,(0) \mathrm{ah}$ \\
\hline C.acutifolum & 4821 & $4,67 a$ & $4,33 \mathrm{ab}$ & $5.00 \mathrm{a}$ & $4.67 \mathrm{a}$ & $4,67 \mathrm{ab}$ & $5,00 \mathrm{a}$ & $3,67 \mathrm{a}$ & $4.67 \mathrm{a}$ \\
\hline C.pubescens & (Test.) & $1.67 \mathrm{de}$ & $2,67 \mathrm{abc}$ & $3,33 \mathrm{abc}$ & 3,67abcd & $1,67 \mathrm{~cd}$ & $3,00 \mathrm{ab}$ & $1.67 \mathrm{a}$ & $2.67 \mathrm{ab}$ \\
\hline C.pubescens & 9229 & $2,00 \mathrm{cde}$ & $2,33 \mathrm{bc}$ & $2.67 \mathrm{abc}$ & $2.33 \mathrm{abcd}$ & $2,00 \mathrm{bcd}$ & $2,67 \mathrm{ab}$ & $2.33 \mathrm{a}$ & $3.33 \mathrm{ab}$ \\
\hline C.pubescens & 16977 & $2,00 \mathrm{cde}$ & $2.33 b c$ & $2.67 \mathrm{abc}$ & $1,67 \mathrm{~cd}$ & $1,33 \mathrm{~cd}$ & $2,00 \mathrm{ab}$ & $1.33 \mathrm{a}$ & $2.33 \mathrm{ab}$ \\
\hline C.pubescens & 17027 & $3,00 \mathrm{abcde}$ & $3,00 \mathrm{abc}$ & $3.33 \mathrm{abc}$ & $3.00 \mathrm{abcd}$ & $1,33 \mathrm{~cd}$ & $2.33 \mathrm{ab}$ & $1.67 \mathrm{a}$ & $2.33 \mathrm{ab}$ \\
\hline C.pubescens & 3191 & $2.67 \mathrm{abcde}$ & $2.67 a b c$ & $2,67 \mathrm{abc}$ & $1.67 \mathrm{ed}$ & $1,00 \mathrm{~cd}$ & $1,67 b$ & $1.67 \mathrm{a}$ & $2.67 \mathrm{ah}$ \\
\hline C.acutifolium & 16926 & $3.00 \mathrm{abcde}$ & $4,00 \mathrm{ab}$ & $4.33 \mathrm{ab}$ & $2.67 \mathrm{abcd}$ & $5,00 \mathrm{a}$ & $4.67 \mathrm{a}$ & $2,67 \mathrm{a}$ & $4.67 \mathrm{a}$ \\
\hline C.pubescens & 17035 & $1,67 \mathrm{de}$ & $2,67 a b c$ & $2.33 \mathrm{abc}$ & $2,00 \mathrm{bcd}$ & $1,33 \mathrm{~cd}$ & $1,67 \mathrm{~b}$ & $1,67 \mathrm{a}$ & $1.67 \mathrm{ab}$ \\
\hline C.pubescens & 9237 & $2,67 \mathrm{abcde}$ & $2.67 \mathrm{abc}$ & $2.33 \mathrm{abc}$ & $1,33 \mathrm{~d}$ & $1,00 \mathrm{~cd}$ & $1,50 \mathrm{~b}$ & $1,00 \mathrm{a}$ & $1,33 \mathrm{ab}$ \\
\hline C.pubescens & 16993 & $1.33 \mathrm{de}$ & $2,33 b c$ & $2.00 \mathrm{bc}$ & $2.33 \mathrm{abcd}$ & $1,50 \mathrm{~cd}$ & $3.00 \mathrm{ab}$ & $2.33 \mathrm{a}$ & $3.00 \mathrm{ah}$ \\
\hline C.acutifolum & 10111 & 3.33abcd & $2,67 a b c$ & 4.00abc & $3,33 \mathrm{abcd}$ & $2,67 \mathrm{abcd}$ & $3.67 \mathrm{ab}$ & $2,33 a$ & $4,00) a b$ \\
\hline \multirow[t]{5}{*}{ C. acutifolıum } & (Test.) & $1,00 \mathrm{e}$ & $1.00 \mathrm{c}$ & 1.67 & $3.67 \mathrm{abcd}$ & $0.67 \mathrm{~d}$ & $2,00 \mathrm{ah}$ & $2.00 \mathrm{a}$ & $1.33 \mathrm{ah}$ \\
\hline & 10227 & & & & & & & & \\
\hline & 9181 & & & & & & & & \\
\hline & 4162 & & & & & & & & \\
\hline & (Test.) & & & & & & & & \\
\hline CV $(\%)$ & & 10,42 & 11,40 & 11,55 & 12,11 & 14,84 & 13,52 & 14,08 & 18,00 \\
\hline
\end{tabular}


MATTOS \& PEDREIRA (1984), observaram produções anuais de matéria seca de Centrosema de $2815 \mathrm{~kg} / \mathrm{ha}$; BUFARAH \& PAULINO (1986), produções de $2587 \mathrm{~kg} / \mathrm{ha}$. Neste experimento a produção média de 1991 foi de $6599 \mathrm{~kg} / \mathrm{ha}$ de matéria seca, e no ano de 1992 foi de 2717 $\mathrm{kg} / \mathrm{ha}$. A TABELA 5 traz os dados de produção de sementes e os meses de florescimento de cada acesso de Centrosema.

SKERMAN (1977) relata que produções de 220 a $275 \mathrm{~kg} / \mathrm{ha}$ de sementes são usuais em Uganda e, $140 \mathrm{~kg} / \mathrm{ha}$, no Norte de Queenslandia e CAMERON (1984) produções de 300 a $600 \mathrm{~kg} / \mathrm{ha}$ obtidas sob irrigação.

A produção de sementes foi pequena em 1989, devido a um florescimento desuniforme e grande quantidade de vagens abortadadas. Os acessos de C.acutifolium praticamente não produziram sementes. As maiores produções do primeiro ano foram obtidas pelos acessos de BRA $10111(88 \mathrm{~kg} / \mathrm{ha})$, e BRA $3191(62 \mathrm{~kg} / \mathrm{ha})$. No segundo ano,os acessos usados como testemunhas, produziram pouca ou nenhuma semente $\mathrm{e}$ os acessos de C.pubescens BRA 16951(494 kg/ha), BRA $16934(374 \mathrm{~kg} / \mathrm{ha})$ e BRA $16942(314 \mathrm{~kg} / \mathrm{ha})$, apresentaram as maiores produções. O florescimento foi mais uniforme ocorrendo nos meses de abril, maio e junho. No terceiro ano, o período de florescimento foi mais longo (abril a setembro), e os acessos com mais produções de sementes, foram: C.pubescens BRA $16942(298 \mathrm{~kg} / \mathrm{ha}), \mathrm{BRA}$ $16918(242 \mathrm{~kg} / \mathrm{ha})$ e BRA $16951(211 \mathrm{~kg} / \mathrm{ha})$.

HERNANDEZ et al. (1992), também verificaram uma grande variabilidade entre acessos quanto a produção de sementes.

A TABELA 6 apresenta o crescimento lateral médio dos acessos de Centrosema. Os acessos que mostraram um maior crescimento lateral, foram: C.acutifolium BRA 9211 e BRA 9229.

$\mathrm{Na}$ TABELA 7 encontram-se as avaliações para a capacidade de enraizamento dos ramos laterais. Os acessos que apresentaram maior enraizamento lateral numa avaliação geral dos resultados descritos na Tabela 7, e, portanto, mostraram hábito rasteiro e mais agressivo, foram: C.acutifolium BRA 9211, BRA 9229 e BRA 9237 e C.pubescens BRA 14419. Por ocasião do $6^{\circ}$ corte, todas as parcelas não apresentaram enraizamento dos ramos laterais.

Verificou-se que os acessos que apresentaram maior crescimento lateral mostraram também a maior produção de matéria seca, o mesmo acontecendo com os acessos que apresentaram maior enraizamento lateral, exceto os acessos C.acutifolium BRA 9237 e C.pubescens BRA 14419.

A TABELA 8 traz a avaliação visual do desenvolvimento dos acessos da Centrosema. Verifica-se, em avaliação geral dos resultados da Tabela 8, que os acessos que apresentaram maior produção de matéria seca (C.acutifolium BRA 9221 e BRA 9229) também se destacaram nas avaliações de desenvolvimento.

\section{CONCLUSÕES}

1. Os acessos que apresentaram a maior produção de matéria seca (média de seis cortes), foram: Centrosema pubescens BRA 16918 (2960 kg/ha); BRA $16985(2720 \mathrm{~kg} / \mathrm{ha})$ e Centrosema acutifolium BRA $9221(2660 \mathrm{~kg} / \mathrm{ha})$; BRA $9229(2610 \mathrm{~kg} / \mathrm{ha})$.

2. Os acessos que mostraram as maiores produções de sementes, foram: C.pubescens BRA $16942 \mathrm{e}$ BRA 16951. O acesso C.acutifolium BRA 9211, apresentou produção razoável de sementes em dois anos e o acesso de C.pubescens BRA 16918, mostrou boa produção de sementes em apenas um ano.

3. Os acessos que tiveram maior crescimento lateral, foram: C.acutifolium BRA 9211 e BRA 9229; e os que apresentaram maior enraizamento lateral, foram: C.acutifolium BRA 9211; BRA 9229; BRA 9237 e C.pubescens BRA 14419.

4. O maior desenvolvimento foi registrado pelos acessos que apresentaram a maior produção de matéria seca: C.acutifolium BRA 9211; BRA 9229.

5. O acesso que apresentou-se mais promissor foi C.acutifolium BRA 9211.

\section{REFERÊNCIAS BIBLIOGRÁFICAS}

BARBOSA-FEVEREIRO, V.P. Centrosema (A.P. de Candolle) Bentham do Brasil - Leguminosae Faboideae. Rodriguesia, v.9, n.42, p.159-219, 1977.

BUFARAH, G.; PAULINO, V.T. Competição de plantas forrageiras no Estado de São Paulo. I. Anhembi. Boletim da Industria Animal, Nova Odessa, v.43, n.1, p.75-85, 1986.

CAMERON, D.G. Tropical and subtropical pastures legumes. 2. Centro (Centrosema pubescens): a legume for the wet tropical coast. Queensland Agricultural Journal, v.110, n.4, p.2221-2225, 1984. 
DELISTOIANOV, J.; PAULINO, V.T.; BUFARAH, G. Competição de plantas forrageiras no Estado de São Paulo. III. Colina, Boletim da Industria Animal, Nova Odessa, v.44, n.1, p.111-123, 1987.

DOBEREINER,J.A.; ARONOVICH,S. Efeito da calagem e da temperatura do solo na fixação de nitrogênio de Centrosema pubescens Benth, em solo com acidez de manganês. IN: CONGRESSO INTERNACIONAL DE PASTAGENS, 9, 1965, São Paulo. Anais. São Paulo: Secretaría da Agricultura do Estado de São Paulo, 1965. 1974p.

HERNANDEZ, B.H.; BOLIVAR, F.D. SCHULTZEKRAFT, R. Adaptacion y productividade de 23 selecciones de Centrosema pubescen Benth en un suelo acido de baja fertilidad del piedemonte Llanero Colombiano. IN: REUNION SABANAS, 1., 1992, Brasilia. Anais. Brasilia: EMBRAPA/CPAC/CIAT, 1992. p.405-13.

KRETSCHMER JR.; A.E. Growth and adaptability of Centrosema species in South Florida. Proceedings of Soil and Crop Science Society of Florida, v.36, p.164-168, 1977.

MATTOS, H.B. Características agronômicas de algumas leguminosas forrageiras de clima tropical. Piracicaba: Escola Superior de Agricultura "Luiz de Queiroz", 1972, 18p. Mimeografado.
MATTOS, H.B.; PEDREIRA, J.V.S. Crescimento estacional de oito leguminosas forrageiras de clima tropical. Boletim da Indústria Animal, Nova Odessa, v.41, n.único, p.145-57, 1984.

MONTEIRO, F.A. Nutrição mineral da Centrosema, Centrosema pubescens Benth. Zootecnia, Nova Odessa,SP, v.15, n.1, p.37-56, 1977.

PAYNE, W.J.A.; LAING, W.I.; MILES, N.S.; MASON, R.R. Fodder and pasture investigational work at Sigatoka, 1949-53. Fiji. Agric. J. v.26, p.38-60, 1955 apud, SKERMAN, P.J. Tropical Forage Legumes, Rome: FAO, 1977, p.244-58 (Plant Production and Protection Series, 2).

SKERMAN,P.J. Tropical forage legumes. Rome: FAO, 1977. 609 p. (Plant Production and Protection, Series No.2).

WILSON, A.S.B.; LANSBURY, T.J. Centrosema pubescens: ground cover and forage crop in cleared rain forest of Ghana. Emp. J. Exp. Agric., v.26, p.351-64, 1958 apud SKERMAN,P.J. Tropical Forage Legumes. Rome:FAO, 1977, 609 p. (Plant Production and Protection Series, 2).

Enviado para publicação em 25.06 .93 Aceito para publicação em 15.09.93 